\title{
Evolution of depression during rehabilitation program in patients with cardiovascular diseases
}

\author{
M. Caru ${ }^{a, b, c}$, D. Curnier ${ }^{b, d}$, M. Bousquet ${ }^{e}$ and L. Kern ${ }^{c, f, g}$
}

${ }^{a}$ Department of Psychology, University of Paris Nanterre, Nanterre, France; ${ }^{b}$ Department of Kinesiology, Laboratory of Pathophysiology of EXercise (LPEX), University of Montreal, Montreal, Canada; 'Laboratoire EA 4430 - Clinique Psychanalyse Developpement (CliPsyD), University

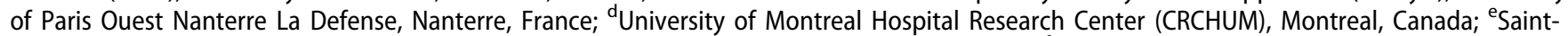
Orens Cardiovascular and Pulmonary Rehabilitation Center, Saint-Orens-de-Gameville, France; fDepartment of Science and Technology of Physical Activities and Sports, University of Paris Nanterre, Nanterre, France; ${ }^{9}$ Laboratoire EA 2931 - Centre de recherches sur le sport et le mouvement (CeRSM), UFR STAPS, University of Paris Nanterre, Nanterre, France

\begin{abstract}
Background: Cardiovascular diseases represent the main cause of death in the world. Rehabilitation through exercise is more and more used in cardiac patients. Given that these patients suffer from depressive symptoms, the risk of having recurrent cardiovascular problems increases. Thus, the aim of this study is to identify the effects of a rehabilitation program on the physiological and psychological parameters; with a particular attention on the depression scores between the scales.

Methods: Twenty-eight cardiac patients participated in this study during a cardiovascular rehabilitation program. They are tested at their entry and at their exit with an evaluation of their physical fitness on an electromagnetic cycle ergometer and by four depression scales (Beck Depression Inventory, Hospital Anxiety and Depression Scale (HADS), Center for Epidemiologic Studies - Depression and Geriatric Depression Screening).

Results: We observe that $21.4-50 \%$ of these patients have depressive symptoms, according to depression scales. The women have depression scores significantly higher than the men. The rehabilitation program improves their maximal oxygen consumption and their maximal aerobic power. At the end of the rehabilitation program, our analysis identifies a significant decrease in the depression score for the HADS. Regardless of the physical deconditioning level and of the improvement of the maximal oxygen consumption, our results show an effect of the rehabilitation program on the depression scores. No correlation between the physical deconditioning and the different depression scores is observed.

Conclusions: This study shows the importance of measuring depression and its severity to improve the care of patients. Our findings show that between $21.4 \%$ and $50 \%$ of patients have depressive symptoms which challenges the categorical approach of the self-report depression scales.
\end{abstract}

\section{> IMPLICATIONS FOR REHABILITATION}

- Depression and cardiovascular diseases have an impact on the patients' physical fitness.

- The rehabilitation program, primarily based around exercise, reduces depressive symptoms.

- As soon as cardiovascular diseases patients enter in a rehabilitation program, the depression should be measured by a psychologist.

- Taking into account the depressive symptoms of the patients as soon as their cardiac event occurs makes it possible to improve the care of patients.
ARTICLE HISTORY

Received 21 November 2017

Revised 8 July 2018

Accepted 9 July 2018

\section{KEYWORDS}

Depression; cardiac rehabilitation; exercise; cardiovascular diseases; depression scales

\section{Introduction}

Cardiovascular diseases (CVDs) represent the main cause of death in the world with 17.5 million deaths each year $(31 \%$ of global mortality) [1]. In Europe, recent data suggest that about half of the deaths are due to CVDs, with almost 4 million of people dying each year [2]. The CVDs are favored by a sedentary population [3] and by the presence of multiple cardiovascular risk factors (i.e., hypertension, obesity, overweight, dyslipidemia, diabetes, smoking, depression) [4].

Depression is an important psychological factor to predict mortality [5] and is known to be associated with CVDs [6,7]. Indeed, depression and anxiety are predictors of coronary heart disease
[6] and of infarcts [7]. Also, depression is a consequence of CVDs since the patients may suffer from a depressive disorder or a severe depression after the development of a heart disease $[8,9]$. Seldenrijk et al. show, in currently depressive patients, a dose-response association between symptom severity and CVDs with a risk of two-fold in a follow-up period of six years [10]. A metaanalysis shows that patients with both depressive symptoms and CVDs have a higher risk of having recurrent cardiovascular problems than patients who have no depressive symptoms [11]. In those with depression without CVDs, physical exercise is associated with a reduction of depressive symptoms [12], which is also observed in depressive patients with CVDs $[13,14]$. The physical 
mechanisms underlying the antidepressant effects of physical activity is in the state of assumptions [12]. Indeed, several physiological and psychological mechanisms of the body could explain the relationship between physical activity and depression (thermogenic hypothesis, endorphin hypothesis, monoamine hypothesis, distraction hypothesis).

Another significant factor in the prognostic of patients with CVDs is their physical fitness [3]. Depression and anxiety are predictors of physical decline in patients with heart failure [15]. Thus, the care of patients has evolved in recent years by integrating physical activity in cardiovascular rehabilitation programs $[16,17]$. Physical activity has been consistently associated with a reduced risk of CVDs [18], a means of prevention against depression [14] and a treatment of depression [19]. Current clinical recommendations include the screening and treating of depression as the "standard of care" for the patient to avoid re-hospitalization within the first 6 months [20]. This is important because the symptoms associated with depression such as fatigue, lack of energy, loss of appetite, sleep problems, psychomotor and attention problems are also associated with a poor prognosis for CVDs outcomes [21].

Several depression scales are used to assess depressive symptoms in patients with CVDs [22,23], allowing the observation of a prevalence depression in people with a heart disease of about $30 \%$ [22]. However, the prevalence of depression is variable within the same scale $[24,25]$. The aim of this study is to identify the effects of a rehabilitation program on the physiological and psychological parameters of CVDs patients; with a particular attention on the depression scores between the scales. Therefore, the first hypothesis is that patients with CVDs will have high depression scores that will differ according to the scales used, while the second hypothesis is that the rehabilitation program will improve the depression score.

\section{Methods}

\section{Participants}

It is a retrospective study where all data are collected in May 2008. Informed consent is obtained from participants. This investigation is conducted in accordance to the World Medical Association Declaration of Helsinki, and is depended upon country rules (law $n^{\circ} 2004-806$; CCTIR 24 October 2005). A total of 28 cardiac patients (22 men and six women) participate in this study consisting of an ambulant cardiovascular rehabilitation program of 4 weeks. The inclusion criteria are all patients with coronary, infarct, heart failure or valvulopathy, whereas the exclusion criteria are applied when the patients are under 18 years old or have a chronic lower respiratory disease. All patients are tested at their entry (time 1) and at their exit (time 2) with at each moment, an evaluation of the physical fitness by an exercise stress test on ergocycle. The patients complete four depression scales. Between time 1 and time 2, they follow a rehabilitation program.

\section{Exercise stress test}

All patients perform an exercise stress test at their entry and at their exit of the rehabilitation program; the tests are performed on an electromagnetic cycle ergometer (Ergoline 900; Bunnik, The Netherlands). A cardiopulmonary exercise test combined with a breath-by-breath combined exercise metabolic and cardiac system (CardiO2; Medical Graphic Corp, St Paul, MN) allows measuring of the maximal aerobic power (MAP) and the maximal oxygen consumption $\left(\mathrm{VO}_{2}\right.$ peak). The exercise stress test is composed of a warm-up at $20 \%$ of the predetermined maximal load followed by an increment of $10 \mathrm{~W}$ per minute.

\section{Depression scales}

The depression scales enable measuring of the depressive symptoms in cardiac patients. This study examines the main depression scales of the scientific literature and especially those studied in the works of Yohannes and Delville [22,23].

- The Beck Depression Inventory (BDI) is used to measure depressive symptoms [26]. It has excellent psychometric properties with Cronbach's alpha coefficient of 0.93 and retests Pearson's $r$ of 0.93 . The BDI is a 21-item multiple-choice self-report inventory for measuring the severity of depression. Statements are reported on a four-points scale ranging from 0 (not at all) to 3 (very much so) in terms of severity; the total score range is of 0-63. All patients are categorized into four groups of depressive symptoms based on standard clinical categories for the BDI. The patients with a score of $0-13$ are categorized as having no depressive symptoms, $14-19$ as mild, 20-28 as moderate and 29-63 as having severe depressive symptoms [27].

- The Hospital Anxiety and Depression Scale (HADS) is used to identify patients with a clinically significant anxiety and depression [28]. The HADS is widely recommended as the screening tool in the cardiac setting [29]. It has excellent psychometric properties with Cronbach's alpha coefficient of 0.85 and Pearson's $r$ of 0.73 . This depression scale is composed of two subscales: an anxiety scale (HADS-A) and a depression scale (HADS-D). Each subscale includes seven-item rated on a four points scale (0-3); a high score indicates more symptoms. Scores for each subscale (anxiety and depression) range from 0 to 21 with scores categorized as follows: no anxiety and depression $0-7$, anxiety and depressive disorders 8-10, severe anxiety and depression 11-21.

- $\quad$ The Center for Epidemiologic Studies - Depression (CES-D) is designed to identify depression among the general population [30]. It has excellent psychometric properties with Cronbach's alpha coefficient of 0.85 and Pearson's $r$ of 0.70 . The CES-D is composed of 20-item, which evaluate various aspects of depressive symptoms: depressed mood, feelings of guilt, hopelessness, psychomotor retardation, anorexia and sleep disorders, according to the previous week. Each answer is rated from 0 (never, rarely - less than a day) to 3 (often, all time - 5-7 days) depending on the frequency of symptoms. A score higher than 17 for men and 23 for women is indicative of depressive disorders.

- The Geriatric Depression Screening (GDS) uses a 30-item selfreport assessment designed to identify geriatric depression [31]. The patients can answer yes or no to the questions depending on the state in which they felt during the previous week. A positive response to 20 of the 30 questions and a negative response to the 10 other questions $(1,5,7,9,15$, $19,21,27,29$, and 30) suggests a possible presence of depression. A score of $0-10$ is represented by the absence of depression, $11-20$ by a mild depression and $21-30$ by a moderate to severe depression. It has excellent psychometric properties with Cronbach's alpha coefficient of 0.94 and Pearson's $r$ of 0.85 .

\section{Rehabilitation program}

The rehabilitation program is especially based around exercise where all patients, within a group, perform exercise training 
during 4 weeks with 5-6 training sessions per week. Each training session is composed of $30-45 \mathrm{~min}$ on a stationary electromagnetic bicycle with a 5-min warm-up at $20 \%$ of the exercise workload and with a 10-min recovery. The exercise workload is determined to entail a heart rate equivalent to a percentage of oxygen uptake (mostly starting at $50 \%$ to attain $80 \%$ at the end of the rehabilitation) prescribed by a cardiologist. The patients perform calisthenics exercises, relaxation, and rest in warm water five times a week to favor the social exchange between patients. Also, therapeutic education sessions are offered in groups of patients for 15-20 min per week. The proposed workshops discuss cardiovascular risk factors, signs and symptoms of the disease, lifestyle, control of stress related to daily management of the disease and exercise (i.e., intensity management, security, benefits).

\section{Statistical analysis}

Statistical analysis is performed using SPSS (SPSS Inc., Chicago, IL). We perform descriptive statistics to describe our population. The quantitative variables are reported as mean \pm standard deviation (SD). A Student $t$-test is used to observe an effect of the rehabilitation program on the physiological and psychological parameters between time 1 and 2. We use an analysis of variance (ANOVA) to assess the effect of the rehabilitation program on depressive symptoms. A Pearson correlation coefficient test is realized to identify a possible relationship between physical deconditioning and the different depression scales. The differences are considered significant when $p$ value is $p<0.05$ with an alpha risk of $5 \%$.

\section{Results}

Our study is composed of 28 patients ( 22 men and six women) with a mean age of $60.11 \pm 11.76$ years, a body mass index of $26.80 \pm 5.54 \mathrm{~kg} \mathrm{~m}^{-2}$, a $\dot{\mathrm{VO}}_{2}$ peak of $18.8 \pm 5.9 \mathrm{ml} \mathrm{kg}^{-1} \mathrm{~min}^{-1}$ and a physical fitness level of $63.3 \%$. Within our population, there are 19 patients with a coronary artery disease (67.9\%), seven patients with a valvulopathy (25\%), one patient with an aneurysm rupture (3.6\%) and one patient with hypertension (3.6\%). We observe, depending on the severity of depression determined by scales (Table 1) in our population, that $50 \%$ (HADS) to $78.6 \%(\mathrm{BDI})$ of patients have no symptoms of depression and that $3.6 \%(\mathrm{BDI})$ to $14.3 \%$ (HADS) have symptoms of severe depression. Overall, we observe that $21.4-50 \%$ of patients have depressive symptoms, according to depression scales.

Table 1. Categorical analysis of the depression in our population by scales.

\begin{tabular}{llrrc}
\hline Scales & \multicolumn{1}{c}{ Categories } & $N$ & \multicolumn{1}{c}{$\%$} & Depressive patients (\%) \\
\hline BDI & No depression & 22 & 78.6 & 21.4 \\
& Mild depression & 5 & 17.9 & \\
& Moderate depression & 0 & 0.0 & \\
& Severe depression & 1 & 3.6 & 25.0 \\
GDS & No depression & 21 & 75.0 & \\
& Mild depression & 5 & 17.9 & \\
& Severe depression & 2 & 7.1 & 28.6 \\
CES-D & No depression & 20 & 71.4 & \\
& Depression & 8 & 28.6 & 50.0 \\
HADS & No depression & 14 & 50.0 & \\
& Depressive disorders & 10 & 35.7 & \\
& Severe depression & 4 & 14.3 & \\
\hline
\end{tabular}

BDI: beck depression inventory; GDS: geriatric depression screening; CES-D: center for epidemiologic studies - depression; HADS: hospital anxiety and depression scale.

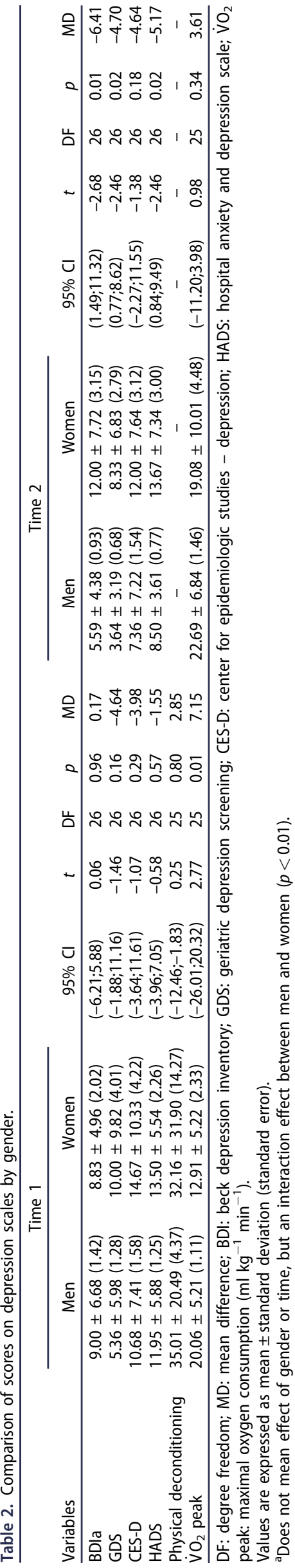


Table 3. Statistical analyses between time 1 and time 2 on different variables studied.

\begin{tabular}{lcccccc}
\hline Variables & Time 1 & Time 2 & $95 \% \mathrm{Cl}$ & $t$ & DF & $p$ Value \\
\hline BDI & $8.96 \pm 6.27(1.18)$ & $6.96 \pm 5.76(1.09)$ & $(-0.19 ; 4.19)$ & 1.87 & 27 & 0.07 \\
GDS & $6.36 \pm 7.03(1.33)$ & $4.64 \pm 4.52(0.85)$ & $(-0.57 ; 4.00)$ & 1.53 & 27 & 0.14 \\
CES-D & $11.54 \pm 8.08(1.53)$ & $8.36 \pm 7.42(1.40)$ & $(-0.15 ; 6.50)$ & 1.96 & 27 & 0.06 \\
HADS & $12.29 \pm 5.74(1.08)$ & $9.61 \pm 4.98(0.94)$ & $(0.92 ; 4.43)$ & 3.13 & 27 & 0.004 \\
MAP & $85.97 \pm 45.75(8.80)$ & $104.89 \pm 53.63(10.32)$ & $(-33.79 ;-4.05)$ & -2.62 & 26 & 0.02 \\
MHR & $109.27 \pm 24.45(4.71)$ & $118.56 \pm 32.51(6.26)$ & $(-23.38 ; 4.80)$ & -1.35 & 26 & 0.19 \\
$\dot{\text { VO }}{ }_{2}$ peak & $18.74 \pm 5.84(1.12)$ & $22.02 \pm 7.43(1.43)$ & $(-5.84 ;-0.73)$ & -2.65 & 26 & 0.01 \\
\hline
\end{tabular}

DF: degree freedom; BDI: beck depression inventory; GDS: geriatric depression screening; CES-D: center for epidemiologic studies depression; HADS: hospital anxiety and depression scale; MAP: maximal aerobic power; MHR: maximal heart rate; $\mathrm{V}_{2}$ peak: maximal oxygen consumption ( $\mathrm{ml} \mathrm{kg}^{-1} \mathrm{~min}^{-1}$ ).

Values are expressed as mean \pm standard deviation (standard error).

Table 4. Pearson's correlation coefficient test between the physical deconditioning and the different depression scales.

\begin{tabular}{|c|c|c|c|c|c|c|c|c|}
\hline \multirow[b]{2}{*}{ Variable } & \multicolumn{2}{|c|}{$\mathrm{BDI}$} & \multicolumn{2}{|c|}{ GDS } & \multicolumn{2}{|c|}{ CES-D } & \multicolumn{2}{|c|}{ HADS } \\
\hline & Time 1 & Time 2 & Time 1 & Time 2 & Time 1 & Time 2 & Time 1 & Time 2 \\
\hline Physical deconditioning & -0.05 & 0.09 & -0.08 & -0.12 & 0.11 & -0.02 & -0.13 & -0.24 \\
\hline
\end{tabular}

BDI: beck depression inventory; GDS: geriatric depression screening; CES-D: center for epidemiologic studies - depression; HADS: hospital anxiety and depression scale.

Table 5. Repeated measures ANOVA's analyses according to improving maximum oxygen consumption ( $\mathrm{V}_{2}$ peak) and physical deconditioning level of patients.

\begin{tabular}{|c|c|c|c|c|c|c|}
\hline Scales & Test & Condition & DF & MS & $F$ & $p$ Value \\
\hline \multicolumn{7}{|c|}{ According to improving maximum oxygen consumption $\left(\dot{\mathrm{V}} \mathrm{O}_{2}\right.$ peak) } \\
\hline \multirow[t]{2}{*}{$\mathrm{BDI}$} & Intra-patients & Time & 1 & 80.42 & 5.60 & \\
\hline & Time $\times \dot{\mathrm{V}} \mathrm{O}_{2}$ peak improvement & 1 & 11.97 & 0.83 & 0.37 & \\
\hline Inter-patients & $\dot{\mathrm{V}} \mathrm{O}_{2}$ peak improvement & 1 & 66.92 & 1.42 & 0.24 & \\
\hline \multirow[t]{3}{*}{ GDS } & Intra-patients & Time & 1 & 45.81 & 2.49 & 0.13 \\
\hline & & Time $\times \dot{\mathrm{V}} \mathrm{O}_{2}$ peak improvement & 1 & 8.03 & 0.44 & 0.51 \\
\hline & Inter-patients & $\dot{\mathrm{V}}_{2}$ peak improvement & 1 & 34.58 & 0.77 & 0.39 \\
\hline \multirow[t]{3}{*}{ CES-D } & Intra-patients & Time & 1 & 184.62 & 5.16 & 0.03 \\
\hline & & Time $\times \dot{\mathrm{V}}_{2}$ peak improvement & 1 & 11.28 & 0.32 & 0.58 \\
\hline & Inter-patients & $\dot{\mathrm{V}} \mathrm{O}_{2}$ peak improvement & 1 & 19.08 & 0.24 & 0.63 \\
\hline \multirow[t]{3}{*}{ HADS } & Intra-patients & Time & 1 & 112.18 & 10.78 & $<0.001$ \\
\hline & & Time $\times \dot{\mathrm{V}} \mathrm{O}_{2}$ peak improvement & 1 & 0.18 & 0.02 & 0.90 \\
\hline & Inter-patients & $\mathrm{V}_{2}$ peak improvement & 1 & 99.73 & 2.34 & 0.14 \\
\hline \multicolumn{7}{|c|}{ According to physical deconditioning level } \\
\hline \multirow[t]{3}{*}{$\mathrm{BDI}$} & Intra-patients & Time & 1 & 76.48 & 5.24 & 0.03 \\
\hline & & Time $\times$ physical deconditioning & 1 & 6.41 & 0.44 & 0.51 \\
\hline & Inter-patients & Physical deconditioning & 1 & 2.64 & 0.05 & 0.82 \\
\hline \multirow[t]{3}{*}{ GDS } & Intra-patients & Time & 1 & 45.00 & 2.41 & 0.13 \\
\hline & & Time $\times$ physical deconditioning & 1 & 1.44 & 0.08 & 0.78 \\
\hline & Inter-patients & Physical deconditioning & 1 & 30.89 & 0.68 & 0.42 \\
\hline \multirow[t]{3}{*}{ CES-D } & Intra-patients & Time & 1 & 182.43 & 5.04 & 0.03 \\
\hline & & Time $\times$ physical deconditioning & 1 & 1.39 & 0.04 & 0.85 \\
\hline & Inter-patients & Physical deconditioning & 1 & 29.78 & 0.38 & 0.54 \\
\hline \multirow[t]{3}{*}{ HADS } & Intra-patients & Time & 1 & 115.19 & 11.57 & $<0.005$ \\
\hline & & Time $\times$ physical deconditioning & 1 & 11.49 & 1.15 & 0.29 \\
\hline & Inter-patients & Physical deconditioning & 1 & 17.44 & 0.38 & 0.54 \\
\hline
\end{tabular}

DF: degree freedom; MS: mean square; BDI: beck depression inventory; GDS: geriatric depression screening; CES-D: center for epidemiologic studies - depression; HADS: hospital anxiety and depression scale.

A significant difference has been shown by gender on the scores of BDI, GDS, and HADS (Table 2). The women have depression scores significantly higher than the men on these three scales at time 2; only CES-D do not show a significant difference. Moreover, depression scores in men seem to decrease at time 2, comparatively to time 1 . Also, at time 1 , men have a $\dot{\mathrm{V}}_{2}$ peak that is significantly higher than women.

The rehabilitation program improves the $\dot{\mathrm{VO}}_{2}$ peak and the MAP (Table 3). HADS have a significant decrease in the depression scores at the end of the rehabilitation program, whereas CES-D and the BDI present a tendency to decrease. No significant improvement of the heart rate at the end of the rehabilitation program is shown.
The results show no correlation between physical deconditioning and the different scores of the depression scales (Table 4).

The patients are separated into two groups according to the median to observe the effect of improving the $\mathrm{V}_{2}$ peak on their depression scores. The group 1 has the lowest improvement of $\dot{\mathrm{V}}_{2}$ peak. The effect of the rehabilitation program according to the improvement of $\mathrm{VO}_{2}$ peak and to each depression scale is visible in Table 5. The repeated measures ANOVA's analyses show an effect of time on intra-patients for the BDI, CES-D, and HADS; no inter-group effect and no interaction effect are observed. The effect of the rehabilitation program according to physical deconditioning and to each depression scale is visible in Table 5. The repeated measures ANOVA's analyses show an effect of time on 
intra-patients for the BDI, CES-D, and HADS; no inter-group effect and no interaction effect are observed.

\section{Discussion}

The aim of this research is to study the physiological and psychological dimensions in patients during a cardiac rehabilitation program. Overall, we observe that patients have improved their depressive symptoms and their physical fitness at their exit of the rehabilitation program, comparatively to their entry.

It is particularly important to target different depression scales in a same studied population because, even if they are standardized tools, there is not a scale that is favorized in comparison to another for older adults [23]. Also, the patients with CVDs have relatively high scores of depression, which seems to differ from one scale to another [22]. According to the categorical approach and the scales used, we observe that between $21.4 \%$ and $50 \%$ of the patients evaluated have depressive symptoms; this observation is in accordance with the literature [22,23]. For example, it is observed in chronic heart failure that the prevalence of depression is high (10-60\% depression) [22]. The sensitivity of these scales in our population may be due to the fact that some are generic while others are specific. Indeed, patients with a cardiac disease have a rate of depression twofold higher than the general population [9,32]. However, according to the scales used, we identify different depression scores from one scale to another. Our results show that between $3.6 \%$ and $14.3 \%$ of patients received a diagnosis of severe depression. These results are important because all scales which examine the prevalence of depression are self-report measures. A patient with a score inferior to $9 / 63$ by the GDS is considered to have no depression. If a patient has a score superior to $10 / 63$, he would then be considered to have a depression ranging from mild to severe. The categorical approach allows the classification of patients by the presence or absence of a trait. Moreover, a patient may be categorized as having symptoms of depression by the GDS, whereas the categorization by the $\mathrm{BDI}$ is reversed. This illustrates that the sensitivity of the depression scales is not yet perfect and deserves to be studied. We note that with the BDI, one on five patients is categorized as being depressive, whereas for the HADS, the depression concerns one of two patients. For the HADS, we observe no consensus on the values of the thresholds used to classify patients. The cutoff for establishing the sensitivity and specificity values is different, according to studies [33,34]. According to the optimal cutoff to screen depression in patients with CVDs, the score in the HADS $\geq 8$ [33], whereas, according to Bjelland et al., the cutoff range is from 4 to 11 [34]. A cutoff too low has for effect an increase in the number of false positives and inversely [35]. Some depression scales overestimate the depression symptoms while other underestimate these symptoms. Our results suggest that the prevalences for the HADS are different from the other three depression scales, which are similar to one another. Our findings show that men have depression scores significantly lower than women after a rehabilitation program (time 2 ) for the BDI and GDS. This is consistent with previous work, which shows that women have significantly higher depression scores on the BDI [36]. This can be explained by the fact that men give more importance to goal-oriented factors (i.e., improvement of $\dot{\mathrm{VO}}_{2}$ peak), whereas women give more importance to interpersonal relationships while internalizing symptoms $[37,38]$.

Regardless of the gender, the rehabilitation program has an effect on the depression score of patients at their exit. Our results show that the practice of physical activity improves the physiological parameters (i.e., MAP and $\mathrm{V}_{2}$ peak) and decreasing the depression scores in patients with CVDs. The improvement in physical fitness level (i.e., $\dot{\mathrm{VO}}_{2}$ peak) is shown to be correlated with improvements of the depression factors [39]. This reduces the deleterious effects associated with the disease because patients with depression and CVDs observe a quicker loss of physical function [8] resulting in the appearance of a physical disability [40]. Indeed, the CVDs patients who have a low functional autonomy have a greater severity of their symptoms and a weaker health-related quality of life, comparatively with CVDs patients without depression $[41,42]$. Nevertheless, contrary to what the literature observes [39], the physical deconditioning level does not influence the depression score between time 1 and time 2 . Indeed, our results show an effect of the rehabilitation program on the depression score, independently of the patient's physical deconditioning level and of the improvement of the $\mathrm{V}_{2}$ peak. Patients who have improved their $\dot{\mathrm{V}}_{2}$ peak the most do not show a significant decrease in their depression scores, compared to patients who have not improved their $\dot{\mathrm{V}}_{2}$ peak. The absence of a control group forces us to remain careful with our results. However, these results may be explained by the social component of our study, which is also the strength of our rehabilitation program. Where most studies put the spotlight on cardiorespiratory response at the expense of psychological responses, our study brings an innovative aspect to current cardiac rehabilitation programs. Indeed, in this study, there is social exchange since the majority of exercise sessions are conducted in a group several times a day. Patients have time to talk and exchange between the activities. The gymnastics activities are an essential component of this rehabilitation program because they consist of short exercise sessions with recovery phases enabling patients to interact with each other more easily. The inclusion of psychological components in cardiac rehabilitation programs should be standard practice by adapted physical activity professionals to improve patient care.

Compared to other studies, which usually take place between 4 and 12 months [43-45], the duration of our program is shorter. Indeed, the duration of the exercise training program is twofold less than other studies [43] and the impact of rehabilitation on improving $\dot{\mathrm{V}}_{2}$ peak and depressive symptoms could be underestimated compared to longer rehabilitation programs. Nevertheless, this does not prevent an improvement in $\dot{\mathrm{V}}_{2}$ peak of $17.5 \%$ in our study. Despite a relatively short rehabilitation program, it is extremely encouraging to observe such effects. Our results are similar to previous studies $[44,46]$ and are higher than others [47]. In the literature, a cardiac rehabilitation program reduces from $20 \%$ to $32 \%$ probability of all-cause mortality [43]. Although these effects are known, many patients often stop the program after a few months of rehabilitation due to external constraints; it is essential to help them become autonomous. The depressive symptoms worsen these aspects, which does not lead to a healthy lifestyle [48]. These results are encouraging in the actual context of patient care which promotes, mostly, the improvement of physiological capacities over the psychological aspects.

\section{Limitations}

Our study identifies some limitations. The first one concerns our number of patients $(n=28)$ which is low compared to other studies in the same field. Moreover, our population is unbalanced regarding the number of men and women, but this did not prevent us from observing interesting differences between them. The 
second one is that we observe in this study a high prevalence between depression scores. Thus, we invite the reader to be more careful in using these depression scales. A diagnosis of depression should not be identified only with one depression scale. In order to have a better diagnosis, it is necessary to couple the depression scales with clinical interviews. Also, it might have been interesting to study the PHQ-9 questionnaire to complete our results. This study being retrospective (data collected in May 2008), we did not know the American Heart Association recommends concerning the PHQ-9 [49]. Finally, patients could have different physical fitness level related to environment (i.e., physical activity), which might vary the response of each patient in the rehabilitation program.

\section{Conclusions}

This study shows the importance of measuring depression and its severity to improve the care of patients. However, our findings show that between $21.4 \%$ and $50 \%$ of the patients have depressive symptoms and that $3.6-14.3 \%$ of patients received a diagnosis of severe depression. The categorical approach which allows classifying patients is questioned. Finally, an effect of the rehabilitation program on both the physiological and psychological parameters is observed but readers should be careful with our results, promote a multidimensional approach and confirm the diagnosis by a psychologist. Following this study, the clinic has a psychologist in their team allowing the establishment of a psychological and physiological follow-up.

\section{Acknowledgements}

We would like to thank the cardiac rehabilitation center, all the staff of service of rehabilitation and all participants of this study. We appreciate the invaluable assistance of Ariane Levesque (McGill University) for her expert review of the article in the English language. The contents of this article are solely the responsibility of the authors and none has received funding to carry out this work.

\section{Disclosure statement}

The authors take responsibility for all aspects of the reliability and freedom from bias of the data presented and their interpretation discussed. The authors report no declarations of interest.

\section{References}

[1] World Health Organization. Global status report on noncommunicable diseases. Geneva: World Health Organization; 2014.

[2] Nichols M, Townsend N, Scarborough $P$, et al. Cardiovascular disease in Europe 2014: epidemiological update. Eur Heart J. 2014;35:2929.

[3] Myers J, McAuley P, Lavie CJ, et al. Physical activity and cardiorespiratory fitness as major markers of cardiovascular risk: their independent and interwoven importance to health status. Prog Cardiovasc Dis. 2015;57:306-314.

[4] World Health Organization. Cardiovascular diseases (CVDs), vol. 2015. Geneva, Switzerland: World Health Organization; 2015.

[5] Kachur S, Menezes AR, De Schutter A, et al. Significance of comorbid psychological stress and depression on outcomes after cardiac rehabilitation. Am J Med. 2016;129:1316-1321.
[6] Kubzansky LD, Cole SR, Kawachi I, et al. Shared and unique contributions of anger, anxiety, and depression to coronary heart disease: a prospective study in the normative aging study. Ann Behav Med. 2006;31:21-29.

[7] Jonas BS, Mussolino ME. Symptoms of depression as a prospective risk factor for stroke. Psychosom Med. 2000;62:463-471.

[8] Gary RA, Dunbar SB, Higgins MK, et al. Combined exercise and cognitive behavioral therapy improves outcomes in patients with heart failure. J Psychosom Res. 2010;69:119-131.

[9] Bounhoure JP, Galinier M, Curnier D, et al. Influence of depression on the prognosis of cardiovascular diseases. Bull Acad Natl Med. 2006;190:1723-1731.

[10] Seldenrijk A, Vogelzangs N, Batelaan NM, et al. Depression, anxiety and 6-year risk of cardiovascular disease. J Psychosom Res. 2015;78:123-129.

[11] Barth J, Schumacher M, Herrmann-Lingen C. Depression as a risk factor for mortality in patients with coronary heart disease: a meta-analysis. Psychosom Med. 2004;66:802-813.

[12] Craft LL, Perna FM. The benefits of exercise for the clinically depressed. Prim Care Companion J Clin Psychiatry. 2004;06:104-111.

[13] Blumenthal JA, Babyak MA, O'Connor C, et al. Effects of exercise training on depressive symptoms in patients with chronic heart failure: the HF-ACTION randomized trial. JAMA. 2012;308:465-474.

[14] Ernstsen L, Rangul V, Nauman J, et al. Protective effect of regular physical activity on depression after myocardial infarction: the HUNT study. Am J Med. 2016;129:82-88.e1.

[15] Shen BJ, Eisenberg SA, Maeda U, et al. Depression and anxiety predict decline in physical health functioning in patients with heart failure. Ann Behav Med. 2011;41:373-382.

[16] Anderson L, Oldridge N, Thompson DR, et al. Exercisebased cardiac rehabilitation for coronary heart disease: Cochrane systematic review and meta-analysis. J Am Coll Cardiol. 2016;67:1-12.

[17] Lavie CJ, Menezes AR, De Schutter A, et al. Impact of cardiac rehabilitation and exercise training on psychological risk factors and subsequent prognosis in patients with cardiovascular disease. Can J Cardiol. 2016;32:S365-S373.

[18] Thompson PD. Exercise and physical activity in the prevention and treatment of atherosclerotic cardiovascular disease. Arterioscler Thromb Vasc Biol. 2003;23:1319-1321.

[19] Milani RV, Lavie CJ, Mehra MR, et al. Exercise training as treatment of depression in heart failure. J Am Coll Cardiol. 2012;59:291.

[20] Lichtman JH, Bigger JT Jr. Blumenthal JA, et al. Depression and coronary heart disease: recommendations for screening, referral, and treatment: a science advisory from the American Heart Association Prevention Committee of the Council on Cardiovascular Nursing, Council on Clinical Cardiology, Council on Epidemiology and Prevention, and Interdisciplinary Council on Quality of Care and Outcomes Research: endorsed by the American Psychiatric Association. Circulation. 2008;118:1768-1775.

[21] Michal M, Wiltink J, Kirschner $Y$, et al. Differential associations of depressive symptom dimensions with cardio-vascular disease in the community: results from the Gutenberg health study. PLoS One. 2013;8:e72014.

[22] Yohannes AM, Willgoss TG, Baldwin RC, et al. Depression and anxiety in chronic heart failure and chronic obstructive 
pulmonary disease: prevalence, relevance, clinical implications and management principles. Int J Geriatr Psychiatry. 2010;25:1209-1221.

[23] Delville $C L, M c D o u g a l l ~ G$. A systematic review of depression in adults with heart failure: instruments and incidence. Issues Ment Health Nurs. 2008;29:1002-1017.

[24] Westlake C, Dracup K, Fonarow G, et al. Depression in patients with heart failure. J Card Fail. 2005;11:30-35.

[25] Freedland KE, Rich MW, Skala JA, et al. Prevalence of depression in hospitalized patients with congestive heart failure. Psychosom Med. 2003;65:119-128.

[26] Beck AT, Steer RA, Brown GK. Beck depression inventory-II. San Antonio (TX): San Antonio, TX: Psychological Corporation; 1996. p. b9.

[27] Beck AT. Depression: clinical, experimental, and theoretical aspects. Philadelphia (PA): University of Pennsylvania Press; 1967.

[28] Zigmond AS, Snaith RP. The hospital anxiety and depression scale. Acta Psychiatr Scand. 1983;67:361-370.

[29] Doyle F, McGee HM, De La Harpe D, et al. The Hospital Anxiety and Depression Scale depression subscale, but not the Beck Depression Inventory-Fast Scale, identifies patients with acute coronary syndrome at elevated risk of 1-year mortality. J Psychosom Res. 2006;60:461-467.

[30] Radloff LS. The CES-D scale a self-report depression scale for research in the general population. Appl Psychol Meas. 1977;1:385-401.

[31] Yesavage JA, Brink TL, Rose TL, et al. Development and validation of a geriatric depression screening scale: a preliminary report. J Psychiatr Res. 1982;17:37-49.

[32] Katon W, Lin EH, Kroenke K. The association of depression and anxiety with medical symptom burden in patients with chronic medical illness. Gen Hosp Psychiatry. 2007;29: 147-155.

[33] Stafford L, Berk M, Jackson HJ. Validity of the Hospital Anxiety and Depression Scale and Patient Health Questionnaire-9 to screen for depression in patients with coronary artery disease. Gen Hosp Psychiatry. 2007;29: 417-424.

[34] Bjelland I, Dahl AA, Haug $\Pi$, et al. The validity of the Hospital Anxiety and Depression Scale. An updated literature review. J Psychosom Res. 2002;52:69-77.

[35] Pittam G, Allaby M. Appraisal of screening for depression: a report for the UK National Screening Committee. UK: UK National Screening Committee; 2014.

[36] Azevedo A, Bettencourt P, Frioes F, et al. Depressive symptoms and heart failure stages. Psychosomatics. 2008;49: 42-48.
[37] Bartels M, Cacioppo JT, van Beijsterveldt T, et al. Exploring the association between well-being and psychopathology in adolescents. Behav Genet. 2013;43:177-190.

[38] Kendler KS, Gardner CO. Sex differences in the pathways to major depression: a study of opposite-sex twin pairs. AJP. 2014;1;171:426-435.

[39] Milani RV, Lavie CJ. Impact of cardiac rehabilitation on depression and its associated mortality. Am J Med. 2007;120:799-806.

[40] Vaccarino V, Kasl SV, Abramson J, et al. Depressive symptoms and risk of functional decline and death in patients with heart failure. J Am Coll Cardiol. 2001;38:199-205.

[41] Jiang W, Kuchibhatla M, Clary $G L$, et al. Relationship between depressive symptoms and long-term mortality in patients with heart failure. Am Heart J. 2007;154:102-108.

[42] Juenger J, Schellberg D, Kraemer S, et al. Health related quality of life in patients with congestive heart failure: comparison with other chronic diseases and relation to functional variables. Heart (Br Cardiac Soc). 2002;87: 235-241.

[43] Taylor RS, Brown A, Ebrahim S, et al. Exercise-based rehabilitation for patients with coronary heart disease: systematic review and meta-analysis of randomized controlled trials. Am J Med. 2004;116:682-692.

[44] Lavie CJ, Milani RV. Benefits of cardiac rehabilitation and exercise training programs in elderly coronary patients. Am J Geriatric Cardiol. 2001;10:323-327.

[45] Kachur S, Chongthammakun V, Lavie CJ, et al. Impact of cardiac rehabilitation and exercise training programs in coronary heart disease. Prog Cardiovasc Dis. 2017;60:103-114.

[46] Lavie CJ, Milani RV. Disparate effects of improving aerobic exercise capacity and quality of life after cardiac rehabilitation in young and elderly coronary patients. J Cardiopulmon Rehabil. 2000;20:235-240.

[47] Jelinek HF, Huang $Z Q$, Khandoker $A H$, et al. Cardiac rehabilitation outcomes following a 6-week program of $\mathrm{PCl}$ and CABG Patients. Front Physiol. 2013;4:302.

[48] Sarris J, O'Neil A, Coulson CE, et al. Lifestyle medicine for depression. BMC Psychiatry. 2014;14:107.

[49] Lichtman JH, Bigger JT, Jr., Blumenthal JA, et al. AHA science advisory. Depression and coronary heart disease. Recommendations for screening, referral, and treatment. A science advisory from the American Heart Association Prevention Committee to the Council on Cardiovascular Nursing, Council on Clinical Cardiology, Council on Epidemiology and Prevention, and Interdisciplinary Council on Quality of Care Outcomes Research. Endorsed by the American Psychiatric Association. Prog Cardiovasc Nurs. 2009;24:19-26. 